\title{
Oxygen-induced circRNA profiles and coregulatory networks in a retinopathy of prematurity mouse model
}

\author{
HUITING ZHOU ${ }^{1}$, HUIHUI SONG ${ }^{2}$, YI WU ${ }^{3}$, XIANG LIU $^{4}$, JING LI $^{4}$, HE ZHAO $^{1}$, MIAOMIAO TANG $^{5}$, \\ XIAOYUAN JI ${ }^{5}$, LU ZHANG ${ }^{5}$, YUANYUAN SU ${ }^{5}$, YAO HE ${ }^{5}$, KEHONG FENG ${ }^{4}$, YANG JIAO ${ }^{6,7}$ and HUA XU ${ }^{4}$ \\ ${ }^{1}$ Institute of Pediatric Research, Children's Hospital of Soochow University, Suzhou, Jiangsu 215025; \\ ${ }^{2}$ Department of Medical Imaging, Suzhou Psychiatric Hospital, The Affiliated Guangji Hospital of Soochow University, \\ Suzhou, Jiangsu 215137; Departments of ${ }^{3}$ Pathology and ${ }^{4}$ Ophthalmology, Children's Hospital of Soochow University, \\ Suzhou, Jiangsu 215025; ${ }^{5}$ Laboratory of Nanoscale Biochemical Analysis, Jiangsu Key Laboratory for Carbon-Based \\ Functional Materials and Devices, Institute of Functional Nano and Soft Materials, Collaborative Innovation Center of \\ Suzhou Nano Science and Technology, Soochow University; ${ }^{6}$ School of Radiation Medicine and Protection, \\ Medical College of Soochow University; ${ }^{7}$ State Key Laboratory of Radiological Medicine and Protection, \\ School of Radiation Medicine and Protection, Soochow University, Suzhou, Jiangsu 215123, P.R. China
}

Received November 15, 2018; Accepted June 27, 2019

DOI: $10.3892 /$ etm.2019.7819

\begin{abstract}
Retinopathy of prematurity (ROP) is a leading cause of childhood blindness. At present, the molecular mechanisms underlying ROP are still far from being clearly understood. Circular RNAs (circRNAs), a novel class of noncoding RNAs, have been reported to serve vital regulatory roles in several human diseases. However, it is still unclear how circRNAs are involved in ROP. In the present study, oxygen-induced retinopathy (OIR) murine retinal samples and paired normal tissues were chosen for high-throughput transcriptome RNA sequencing and bioinformatic analyses. As a result, a total of 236 differentially expressed circRNAs, 14 differentially expressed miRNAs, and 9,756 differentially expressed mRNAs were identified in the OIR samples. Gene ontology analysis showed that angiogenesis ranked in the top five upregulated biological processes associated with differential mRNA expression. Then, 66 co-expression pairs of circRNA-mRNA were predicted according to the mRNAs that were enriched in angiogenesis. Furthermore, coregulation prediction was separately performed to identify the differentially expressed miRNAs that targeted angiogenesis-associated circRNAs or
\end{abstract}

Correspondence to: Dr Hua Xu, Department of Ophthalmology, Children's Hospital of Soochow University, 92 Zhongnan Road, Suzhou, Jiangsu 215025, P.R. China

E-mail: xuhua01431@suda.edu.cn

Dr Yang Jiao, School of Radiation Medicine and Protection, Medical College of Soochow University, 199 Renai Road, Suzhou, Jiangsu 215123, P.R. China

E-mail: jiaoyang@suda.edu.cn

Key words: retinopathy of prematurity, oxygen-induced retinopathy, circular RNA, RNA sequencing, angiogenesis
mRNAs. Finally, nine differentially expressed circRNAs were predicted to be competing endogenous RNAs by constructing a circRNA-miRNA-mRNA network followed by reverse transcription-quantitative PCR validation. The results of the present study suggest that the identified set of circRNA transcripts and the potential regulatory mechanisms for the development of ROP are worthy of functional studies.

\section{Introduction}

Retinopathy of prematurity (ROP) is a vasoproliferative disease that primarily occurs in premature babies and in infants with very low birth weights. Retinopathy leads to a series of sequelae, including ametropias, strabismus, amblyopia, complicated cataracts, secondary glaucoma, retinosis, retinal detachment and even blindness $(1,2)$. It has been shown that the administration of high concentrations of oxygen, which is primarily administered to improve the survival of preterm infants, is associated with ROP, even as early as the first wave of ROP (3). Oxygen toxicity caused by dangerously high oxygen saturations (phase 1) followed by hypoxia-mediated vasoproliferation (phase 2) was established and verified in both animal and human studies (4-7). It is well recognized that the hyperoxia-induced suppression of oxygen-regulated angiogenic growth factors, such as erythropoietin (8) and vascular endothelial growth factor (VEGF) (9), leads to both the arrest of retinal vessel growth and the partial loss of existing retinal vessels (phase 1) (10). Subsequently, the increased metabolic requirements and the attenuated vasculature of the developing retina lead to retinal hypoxia, which compensates by stimulating growth factor-induced vasoproliferation (phase 2); finally, this causes fibrous scar formation, retinal detachment, and blindness (1).

At present, intraocular anti-VEGF therapies and several clinical therapeutics, including surgery, cryotherapy and laser photocoagulation, have effectively improved the treatment outcome of ROP $(11,12)$. Furthermore, numerous studies have 
ascertained the pathophysiological mechanisms underlying ROP, such as structural and functional anomalies, oxidant stress and the roles of growth factors, including VEGF and insulin-like growth factor (12). Nevertheless, knowledge is still lacking about the specific molecular mechanisms, which underlie vasculogenesis and angiogenesis during ROP.

Strategies such as high-throughput techniques and bioinformatics analyses have also been widely used to investigate ROP mechanisms. For example, as early as 2009 , Sato et al (13) revealed the gene expression profile of an oxygen-induced retinopathy (OIR) murine model, a valuable platform used to adequately reproduce the vaso-obliteration and neovascularization phases of ROP (5). Thereafter, several bioinformatics predictions and functional studies focused on microRNAs (miRNAs), an important subtype of small noncoding RNAs that are potential critical regulators during retinal vascular angiogenesis in ROP (14-21).

Currently, a novel class of RNA transcripts named circular RNAs (circRNAs) have emerged as potential regulators in a number of different biological processes and various diseases; they primarily serve as miRNA 'sponges' that naturally sequester and competitively suppress miRNA activity $(22,23)$. Recently, a few studies have reported the involvement of circRNAs in retinal development and in several other ocular diseases using a high-throughput sequencing analysis (24-26). For example, Han et al (24) identified the circRNA profile during the postnatal development of rat retinas by RNA sequencing and indicated the association between circRNAs and physiological neuronal apoptosis in the developing nervous system. Singh et al (25) analyzed 74 differentially expressed circRNAs from the 12,532 circRNAs identified in the eyes of cystathionine- $\beta$-synthase-deficient mice and identified several miRNAs that are specific to each differential circRNA, suggesting a novel regulatory mechanism in hyperhomocysteinemia-induced ocular disorders. Luo et al (26) identified the alanine, aspartate and glutamate metabolic pathway as a target for microRNA (miR)-184 and predicted the expression patterns of circRNAs in the miR-184-transfected human lens epithelial cell line to provide a novel avenue for understanding the role of miR-184 in ocular diseases. Nevertheless, the expression profiles and regulatory networks of circRNAs in ROP remain largely unknown.

In the present study, a high-throughput RNA sequencing strategy and bioinformatics analysis were used to examine the circRNA profiles and the interaction of the circRNA-miRNA-mRNA networks in OIR mouse retinas. The results of the present study predicted a novel regulatory role of circRNA in RNA-RNA coregulation in OIR, which may provide novel insights into the potential mechanisms underlying the development of ROP.

\section{Materials and methods}

Animals. C57BL/6J timed-pregnant dams $(2.5 \pm 0.5$ months old; weight $33 \pm 0.2 \mathrm{~g} ; \mathrm{n}=6$ ) were purchased from Nanjing SIKERUI Biological Technology Co., Ltd. (Nanjing, China). The mice were kept in specific, pathogen-free conditions at the Animal Center of Soochow University (Suzhou, China), housed at $23 \pm 2^{\circ} \mathrm{C}$ and $50 \pm 10 \%$ humidity with a 12 -h light/dark cycle with free access to food and water. The animals were treated in accordance with the ARVO Statement for the Use of Animals in Ophthalmic and Vision Research (27). All procedures employed for the animal experiments in the present study were approved by the Animal Care and Use Committee of Soochow University.

Oxygen-induced retinopathy newborn mouse model. A total of 20 neonatal mice ( 8 female and 12 male; weight $3 \pm 0.5 \mathrm{~g}$ ) were used in the following study and were housed in the same conditions as the pregnant dams. An OIR newborn mouse model of ROP was constructed as described previously (5). Briefly, 7-day-old healthy neonatal mice were randomly divided into the OIR group $(n=10)$ or the control group $(n=10)$. For OIR model construction, 7-day-old mice and their nursing mothers were maintained in $75 \%$ oxygen for 5 continuous days. On postnatal day (P) 12, the pups were returned to normoxic air and kept with their mothers for another 5 days. During the entire procedure, the age-matched mouse pups were kept in normoxic air as the control group. On P17, the mouse pups and their mothers were anaesthetized with $10 \%$ chloral hydrate $(300 \mathrm{mg} / \mathrm{kg}$ body weight; Sigma-Aldrich; Merck KGaA, Darmstadt, Germany) via intraperitoneal injection (14) and were sacrificed by cervical dislocation. The retinas of pups were collected for pathology determination and RNA extraction. During the entire experimental process, there were no signs of peritonitis observed in both the newborn pups and the adult mice. The present study was approved by The Ethics Review Board of The Children's Hospital of Soochow University (Suzhou, China).

Retina isolation and vascular staining. On P17, the mouse pups ( $\mathrm{n}=4$ /group) were anaesthetized with $10 \%$ chloral hydrate ( $300 \mathrm{mg} / \mathrm{kg}$ body weight; Sigma-Aldrich; Merck KGaA) via intraperitoneal injection and were sacrificed by cervical dislocation. The intact ocular globe was enucleated and fixed with $4 \%$ paraformaldehyde for 1-h at room temperature. After being washed twice in $1 \mathrm{X}$ PBS, the retinas were carefully separated from lens and vitreous under a light dissection microscope (Olympus Corporation, Tokyo, Japan; magnification, x20). The retinas were subsequently subjected to isolectin B4-594 (cat. no. I21413; Alexa Fluor; Molecular probes, Eugene, OR, USA) staining at $4^{\circ} \mathrm{C}$ overnight, as described previously (6) and were cut into four quadrants and mounted onto a slide. The staining results were determined and imaged under a fluorescence microscope (Olympus Corporation, Tokyo, Japan; magnification, x40). Retinal whole-mounts were continuously imaged and stitched together using Adobe Photoshop CS5 (Adobe Systems Europe, Ltd., Maidenhead, UK).

RNA library preparation, whole transcriptome RNA and small RNA sequencing. The total RNAs from retinas of mice with OIR and normoxic controls on P17 ( $\mathrm{n}=3$ /group) were extracted using TRIzol ${ }^{\circledR}$ Reagent (Invitrogen; Thermo Fisher Scientific, Inc., Waltham, MA, USA) and the strand-specific RNA-seq libraries were constructed using TruSeq Stranded Total RNA with Ribo-Zero Gold kit (Illumina, Inc., San Diego, CA, USA) according to the manufacturer's instructions as described previously (28). A $1 \mu \mathrm{g}$ aliquot of each RNA sample was used for library construction. Briefly, after the removal of the ribosomal RNA using the Ribo-Zero rRNA Removal kit 
(Illumina, Inc.), the RNA was purified by ethanol precipitation and was fragmented. RNA fragmentation was performed using divalent cations at $94^{\circ} \mathrm{C}$ in $5 \mu \mathrm{l}$ rRNA Binding Buffer and $5 \mu \mathrm{l}$ rRNA Removal Mix-Gold. First strand cDNA was synthesized using $8 \mu \mathrm{l}$ First Strand Synthesis Act D Mix and SuperScript II Reverse Transcriptase at $25^{\circ} \mathrm{C}$ for $10 \mathrm{~min}, 42^{\circ} \mathrm{C}$ for $15 \mathrm{~min}$ and $70^{\circ} \mathrm{C}$ for $15 \mathrm{~min}$. Second strand cDNA was synthesized using $5 \mu 1$ End Repair Control and $20 \mu 1$ Second Strand Marking Master Mix. The double strands cDNA was purified by AMPure XP beads (Beckman Coulter, Inc., Brea, CA, USA). After adenylate $3^{\prime}$ ends and adapters ligation, library fragments were purified using the AMPure XP system (Beckman Coulter) for selecting cDNA fragments of preferentially 150-200 bp in length. DNA fragments was enriched through PCR process, and the PCR mixture was incubated in following progress: 1 cycle $98^{\circ} \mathrm{C}$ for $30 \mathrm{sec} ; 15$ cycles $98^{\circ} \mathrm{C}$ for $10 \mathrm{sec}$, $60^{\circ} \mathrm{C}$ for $30 \mathrm{sec}, 72^{\circ} \mathrm{C}$ for $30 \mathrm{sec} ; 1$ cycle $72^{\circ} \mathrm{C}$ for $5 \mathrm{~min}$; hold at $10^{\circ} \mathrm{C}$. Finally, products were purified (Agencourt AMPure $\mathrm{XP}$, Beckman Coulter) and library quality was assessed on the Agilent Bioanalyzer 2100 system (Agilent Technologies, Inc., Santa Clara, CA, USA). The libraries were sequenced on the Illumina HiSeq X-Ten sequencing platform (OE Biotech, Inc., Shanghai, China, http://www.oebiotech.com/), 150 bp paired-end reads were generated. For small RNA sequencing, the total RNA was ligated sequentially with 3 ' and 5' adaptors, and RT-PCR was performed using the TruSeq ${ }^{\mathrm{TM}}$ Small RNA Sample Prep kits (Illumina, Inc.). After isolation using a 6\% Tris-borate-EDTA-PAGE gel, the resulting ligation products were sequenced using the same sequencing platform.

Identification and analysis of OIR-responsive differential circRNAs and miRNAs. circRNAs were predicted using CIRI software (v.2.0.3), as described by Memczak et al (22) and Gao et al (29). Briefly, the clean reads were aligned to the reference genome using Bowtie2 (http://bowtie-bio.sourceforge. net/bowtie2/manual.shtml); for the unmapped reads, the junctions were picked out using a back-splice algorithm in CIRI software (v.2.0.3). The total circRNAs were blasted against the mouse circRNAs in the circBase database (30) to identify the total novel circRNAs and the total known circRNAs. The expression level of the circRNAs was measured by mapped back-splicing junction reads per million mapped reads. To screen out the differentially expressed circRNAs in OIR retinas, DESeq software (v.1.18.0) (31) was used to normalize the junction read counts of circRNA for each sample, calculate the fold change (FC) and analyze the differential significance between the OIR and control groups. A FC $>2$ and $\mathrm{P}<0.05$ were selected as significance thresholds.

In addition, the primary functional annotation was performed according to the annotation of circRNA-originated coding genes. Briefly, Gene Ontology (GO) analysis was performed using the DAVID (6.8) online tool (https://david. ncifcrf.gov/) (32) by calculating the number of circRNAs enriched for each GO item. The significance of circRNA enrichment in each GO item was calculated by a hypergeometric distribution test. To explore the pathways possibly regulated by differential circRNAs, a Kyoto Encyclopedia of Genes and Genomes (KEGG) analysis was performed to determine the most commonly enriched cellular pathways (33). In addition, miRanda (34) software (v.3.3a) was used to predict the miRNAs that may be sequestered by differential circRNAs that were acting as sponges, and the circRNA-miRNA target interaction network was plotted using the R network package (v.3.6.0, http://www.R-project.org/) (35). Small RNA sequencing was performed and the clean reads were obtained by removing the low-quality reads, the reads with $5^{\prime}$ primer contaminants and poly (A) tails, the reads without the $3^{\prime}$ adapter and insert tag, and the reads shorter than $15 \mathrm{nt}$ and longer than $41 \mathrm{nt}$. Subsequently, RNA were subjected to the BLAST search against the Rfam v.10.1 (http://www.sanger. ac.uk/software/Rfam) (36) and GenBank (http://www.ncbi. nlm.nih.gov/genbank/). The known miRNAs were identified by aligning against miRBase v.21 databases (http://www.mirbase. org/) (37) and the unannotated small RNAs were analyzed by mirdeep2 (38) to predict novel miRNAs. Differentially expressed miRNAs were identified with a threshold of $\mathrm{P}<0.05$, which was calculated with the DEG algorithm (v.1.18.0).

Prediction of competing endogenous circRNA (ceRNA) and ceRNA networks (ceRNETs) in the OIR model. To examine the potential regulatory roles of circRNAs serving as ceRNA via the circRNA-miRNA-mRNA network in ROP: i) Differential mRNAs and miRNAs from the same OIR samples were separately identified based on whole transcriptome RNA and small RNA sequencing, followed by bioinformatics analysis (Shanghai OE Biotech, Co., Ltd., Shanghai, China); ii) the GO enrichment analysis of the differentially expressed mRNAs was performed; iii) the correlation between miRNA-target (miRNA-mRNA or miRNA-circRNA) was predicted using the miRanda algorithm, with the following parameters: $\mathrm{S} \geq 150, \Delta \mathrm{G} \leq-30 \mathrm{kcal} / \mathrm{mol}$ and a strict $5^{\prime}$ seed pairing (39); iv) the ceRNA prediction was performed via base sequence and expression value analysis based on the ceRNA_score formula (ceRNA_score = MRE_for_share_miRNA/MRE_ for_circRNA_miRNA); and v). Finally, the ceRNETs were constructed and mapped using Cytoscape software (version 3.2.1, https://cytoscape.org/). Pearson's correlation coefficient and P-values were calculated based on the expression levels of mRNAs, miRNAs and circRNAs, a Pearson's correlation coefficient $\geq 0.7$ and a P-value $\leq 0.05$ were selected as the correlation analysis parameters, and a $\mathrm{P}$-value $\leq 0.05$ was used as the ceRNA analysis parameter.

Reverse transcription-quantitative polymerase chain reaction $(R T-q P C R)$. To validate the reliability of high-throughput RNA sequencing and explore the expression trends of circRNA, miRNA and mRNA, RT-qPCR was performed using Q-SYBR green Supermix (Bio-Rad Laboratories, Inc., Hercules, CA, USA) on an Applied Biosystems 7500 FAST Real-Time PCR system (Applied Biosystems; Thermo Fisher Scientific, Inc.). The total RNA from the retinas of mice with OIR and normoxic controls on P17 ( $n=3 /$ group) were extracted using TRIzol ${ }^{\circledR}$ Reagent (Invitrogen; Thermo Fisher Scientific, Inc.) according to the manufacturer's protocol (40). First-strand cDNA was synthesized from $2 \mu \mathrm{g}$ RNA per sample using a Transcriptor First Strand cDNA Synthesis kit (Roche Diagnostics, Indianapolis, IN, USA) with the reaction conditions were $10 \mathrm{~min}$ at $65^{\circ} \mathrm{C}, 30 \mathrm{~min}$ at $55^{\circ} \mathrm{C}$ and $5 \mathrm{~min}$ at $85^{\circ} \mathrm{C}$. For RT-qPCR, denaturation was performed at $95^{\circ} \mathrm{C}$ for $15 \mathrm{~min}$ followed by 40 cycles of $95^{\circ} \mathrm{C}$ for $10 \mathrm{sec}, 60^{\circ} \mathrm{C}$ 
Table I. Primer sequences for reverse transcription-quantitative polymerase chain reaction.

\begin{tabular}{ll} 
Gene symbol & \multicolumn{1}{c}{ Forward primer, 5'-3' } \\
\hline mmu-miR-203-3p & GTGAAATGTTTAGGACCACTAG \\
mmu_circ_0013414 & TACAGACAGTGACAGAGAAGCA \\
mmu_circ_0012698 & CGGCCTTATCTGACCTGTAT \\
mmu_circ_0005477 & CAAGGTTTCCTCCAAGAGATG \\
mmu_circ_0000668 & CCAACCATTGAGCAAATGAAAG \\
Srpk2 & CATTGCCTTGCTTATTACCCT \\
Rtn4 & GGCATCAGGCGCAGATAGAT \\
Ptprb & TGGATGTTTGGTTGTACCTACT \\
Nrp1 & TCAGCTCTGTTTACCAAGCA \\
Mapk14 & TACTTTGCTCAGTACCACGAC \\
Kdr & TGGGATGAAATCTTTGGTGGA \\
Hmox1 & CAAGTTCAAACAGCTCTATCGT \\
Hif1a & TCTGTTCCCATTAGCAGGTGA \\
Fn1 & ACCACCTCTATCCTCATGT \\
Flna & GGTCTCAGTCAAGTTCAACG \\
Efnb2 & GCCAAATCCAGGTTCTAGC \\
Cav1 & GTGCTCAGCTATGTAGCATC \\
GAPDH & ACCACAGTCCATGCCATCAC \\
U6 & CTCGCTTCGGCAGCACA
\end{tabular}

Reverse primer, 5'-3'

$\begin{array}{lr}\text { GCTGTCAACGATACGCTACGTAACG } & 22 \\ \text { GGCCCATGATTATTCAGAGG } & 109 \\ \text { GCAACTGCATCTAATGGTCT } & 106 \\ \text { GGTCACTTCGCTTCGTTC } & 122 \\ \text { GGAAGAGAGAATGCTGTGGA } & 108 \\ \text { CCTTTAGCTTTGTTGACACTAC } & 81 \\ \text { CCGTAACCAAGGGAGTGTCC } & 165 \\ \text { GATCTATTACCCTTCACACACG } & 84 \\ \text { ATGGAGGTCACAAACACGA } & 83 \\ \text { AGGCTCTTCCACTCATCTAT } & 101 \\ \text { ATATCAGGAGCTGGGTAACT } & 84 \\ \text { GGTCTTAGCCTCTTCTGTCAC } & 88 \\ \text { AGATTCTGACATGCCACATAG } & 86 \\ \text { CAGAACTCTCCTGGAACG } & 105 \\ \text { ACCCTGACTCCTGAAGACTA } & 116 \\ \text { TGCGATCCCTGCGAATAAG } & 94 \\ \text { CAGTCCAGAGAGGTCTCAC } & 102 \\ \text { TCCACCACCCTGTTGCTGTA } & 452 \\ \text { AACGCTTCACGAATTTGCGT } & 94\end{array}$

miR, microRNA; circ, circulating RNA; Srpk2, SRSF protein kinase 2; Rtn4, reticulon 4; Ptprb, protein tyrosine phosphatase receptor type B; Nrp1, neuropilin 1; Mapk14, mitogen-activated protein kinase 14; Kdr, kinase insert domain receptor; Hmox1, heme oxygenase 1; Hif1a, hypoxia-inducible factor 1 subunit alpha; Fn1, fibronectin 1; Flna, filamin alpha; Efnb2, ephrin B2; Cav1, caveolin 1; GAPDH, glyceraldehyde-3-phosphate dehydrogenase; U6, U6 small nuclear RNA.

for $20 \mathrm{sec}$ and $72^{\circ} \mathrm{C}$ for $30 \mathrm{sec}$ using SuperReal PreMix Plus SYBR Green (Tiangen Biotech, Co., Ltd., Beijing, China). Certain differential circRNAs, miRNAs and mRNAs associated with angiogenesis were determined, and the expression of RNAs were based on the quantification cycle value $(\mathrm{Cq})$ and the relative expression levels were calculated using the $2^{-\Delta \Delta \mathrm{Cq}}$ method (41). GAPDH and U6 served as internal standard controls, and all reactions were performed in triplicate. The primers used are shown in Table I.

Statistical analysis. All data are presented as the mean \pm standard deviation. A student's t-test was used to analyze the significant difference in the expression of circRNA, miRNA and mRNA between OIR and normoxic control groups and Pearson's rank correlation analysis was used for the correlation analysis in ceRNA networks. The statistical tests were performed using SPSS version 19.0 (IBM, Corp., New York, NY, USA). P $<0.05$ was considered statistically significant.

\section{Results}

Overview of the sequencing results and identification of circRNAs in OIR mouse retinas. OIR mice is a widely accepted model of ROP as the retinas in the model mice are very similar to those of premature infants with regard to the highly immature retinal vessel structure which is hypersensitive to hypoxia damage (42). In the present study, 7-day-old (P7) mouse pups

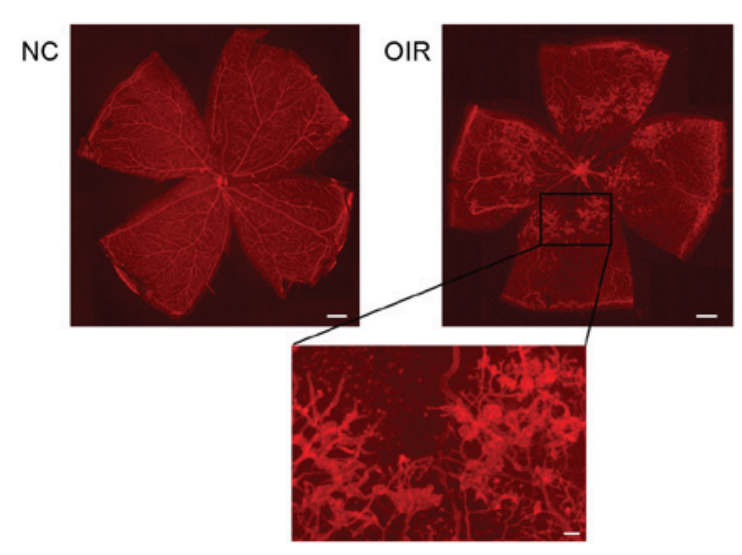

Figure 1. Visualization of OIR in P17 mice. Retinal whole-mounts in the P17 $\mathrm{NC}$ and OIR groups are shown by isolectin B4-594 fluorescent dye staining. Inset, the dysregulated neo-vessels in the OIR group were locally magnified. Scale bar, $10 \mu \mathrm{m}$ in the retinal whole-mounts and scale bar, $1 \mu \mathrm{m}$ in the magnified inset. NC, negative control; OIR, oxygen-induced retinopathy.

were separately exposed to $75 \%$ oxygen or normal air from P7 to P12 to inhibit retinal vessel growth and to cause significant vessel loss. On P12, the mice were subsequently returned to normoxic conditions for 5 days. As expected, both retinal neovascularization and normal vessel regrowth driven by the hypoxic avascular retina manifested on P17, as shown in Fig. 1. For example, obvious pathological retinal angiogenesis, such 
A circRNA length distribution

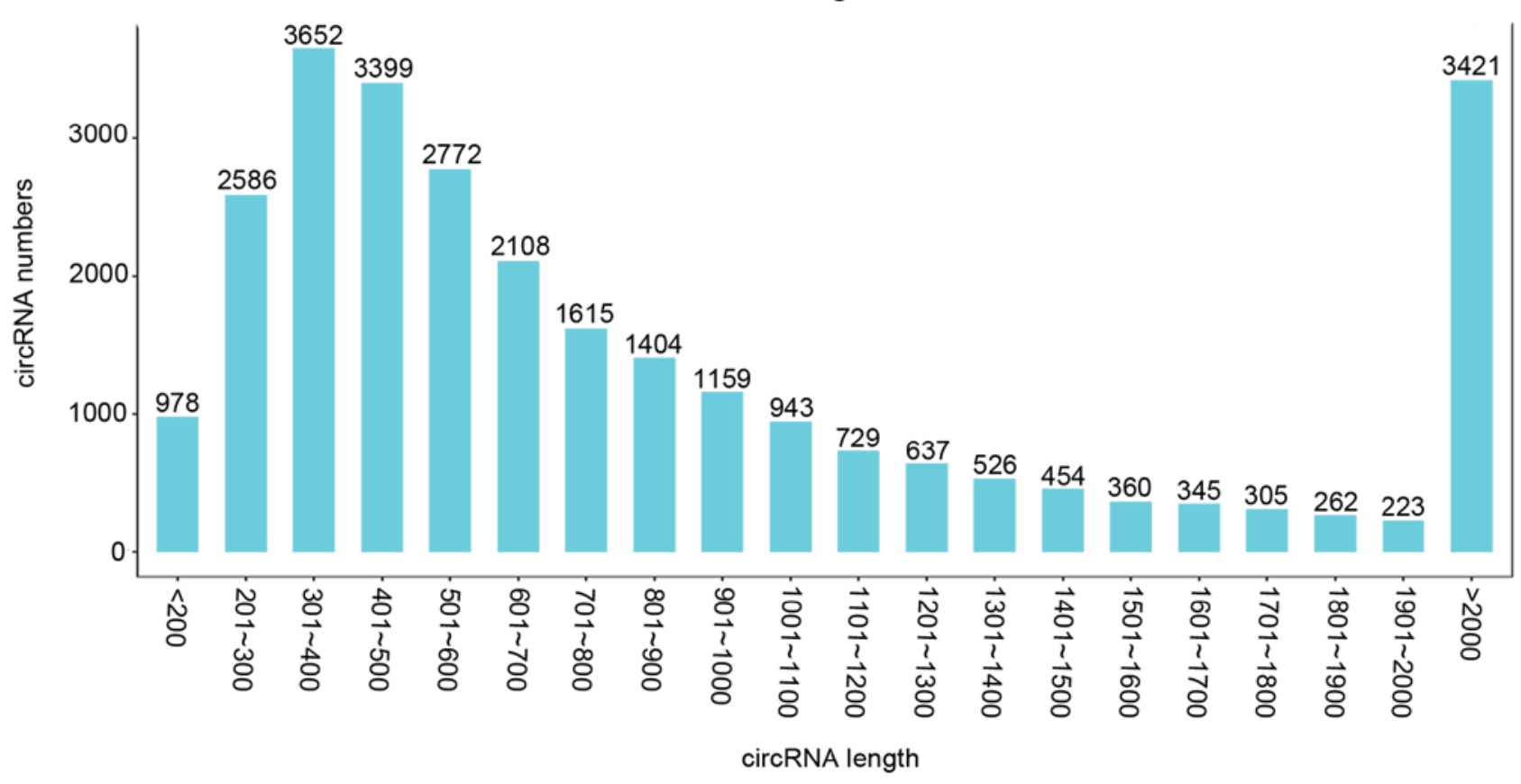

B

Exon per circRNA

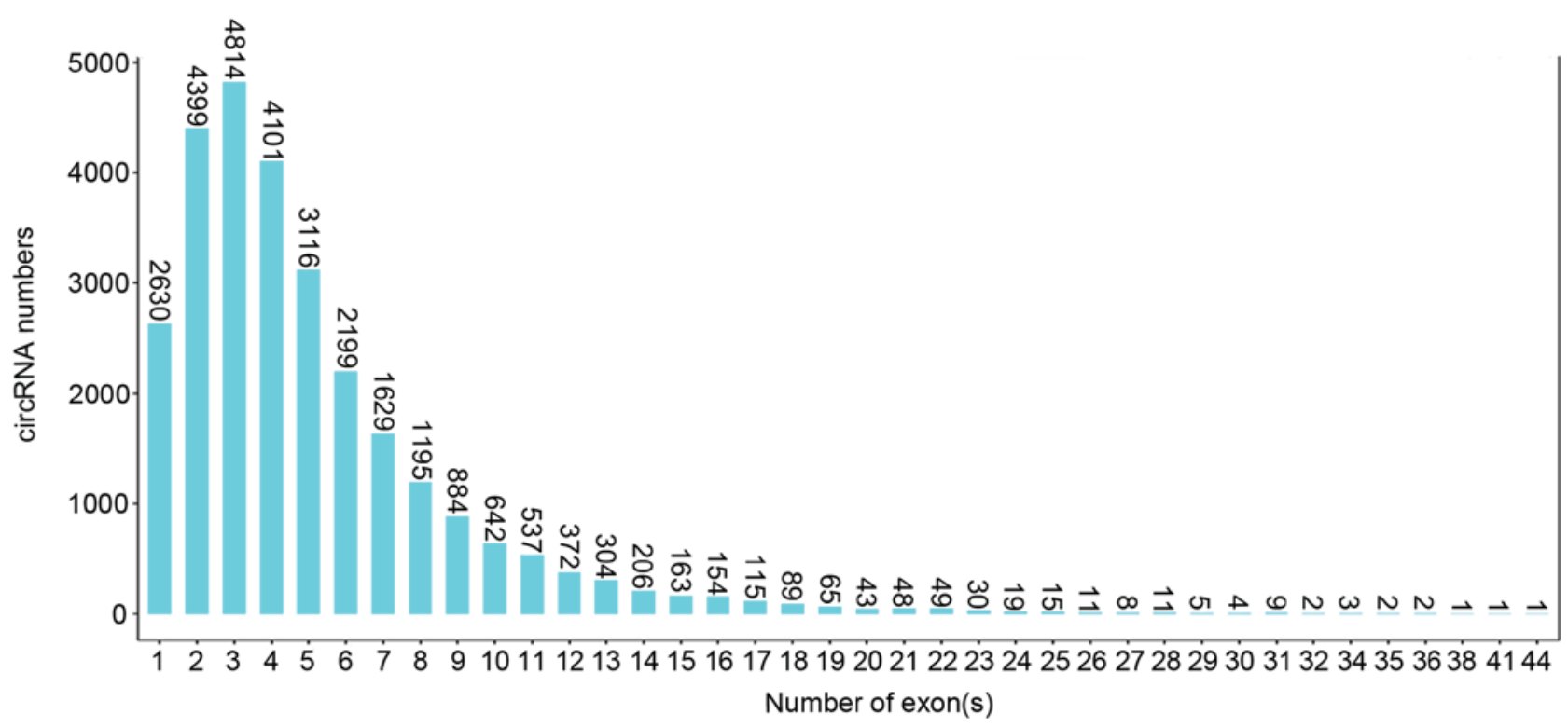

Figure 2. Analysis of the genome features of the predicted circRNAs. (A) Length distribution and (B) exon number distribution of the predicted circRNAs were analyzed. circRNA, circulating RNA.

as uneven and disorganized retinal vessels with obvious dilatation and tortuosity was observed in OIR newborn mice on P17 compared with the control group.

To investigate whether circRNAs was involved in ROP, the extracted RNAs from the mouse retinas were first subjected to high-throughput transcriptome RNA sequencing on P17, and 27,878 candidate circRNAs were identified. Of all the candidates, 18,695 (67.1\%) had a length between 200 and 1,000 bp (Fig. 2A), 19,060 (68.4\%) had no more than 5 exons (Fig. 2B), and all candidate circRNAs were found to be distributed among all the chromosomes (Fig. 3A). According to the genomic loci of these circRNAs, 25,583 $(91.77 \%)$ candidates were categorized as sense-overlapping circRNAs, 1,010 (3.62\%) were determined as exonic circRNAs, 768 (2.75\%) candidates were found to be intergenic, $326(1.17 \%)$ were antisense circRNAs, and $191(0.69 \%)$ were identified as intronic circRNAs (Fig. 3B). In addition, 7,767 (27.9\%) circRNAs were identified as known circRNAs by performing a BLAST search against the known mouse circRNAs in the circBase database (Fig. 3C).

Functional annotation of differential circRNAs and mRNAs in the OIR model. To identify the possible roles of circRNAs in ROP, the 236 differentially expressed circRNAs, 67 upregulated and 169 downregulated circRNAs, in OIR retinas were screened using the DESeq software (31) (Fig. 4A and Table SI) and were functionally annotated. Using GO analysis, the 
A circRNA distribution on chromosome/scaffold

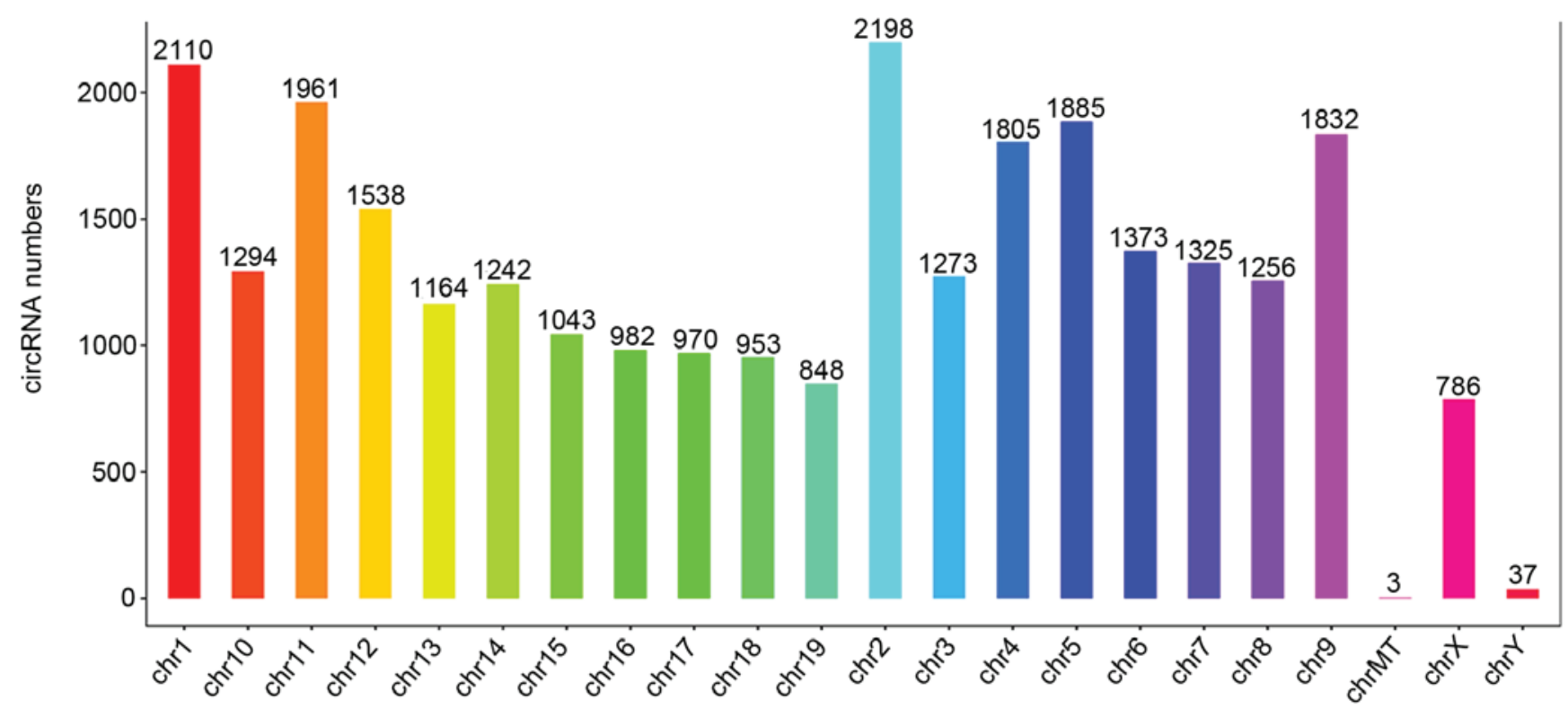

B
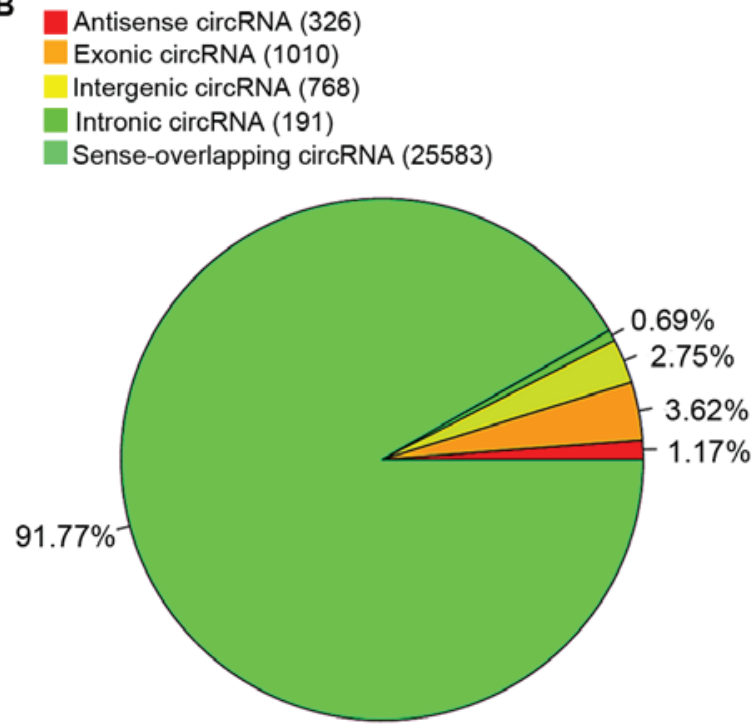

\section{Chromosome or scaffold}

C

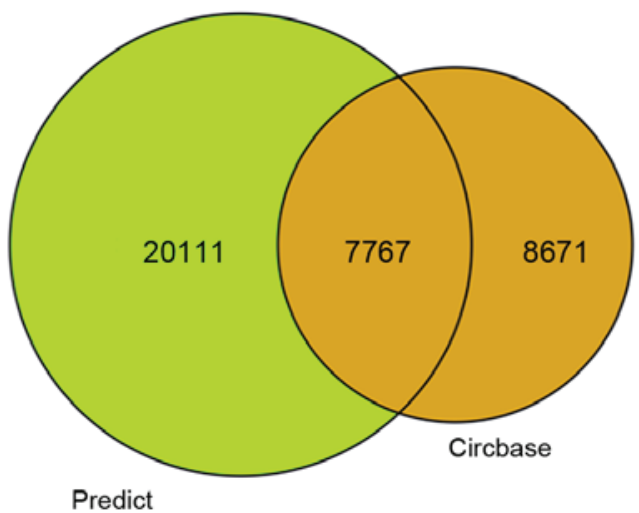

Figure 3. Additional genome features of the predicted circRNAs. (A) Chromosome distribution, (B) subgroup classification and (C) known circRNA identification via circBase of the predicted circRNAs were also analyzed. circRNA, circulating RNA; chr, chromosome.

differential circRNAs were shown to be primarily enriched in the biological processes of the positive regulation of cholesterol esterification, very-low-density lipoprotein particle remodeling and lipoprotein metabolic processes (Fig. 4B and Table II). In addition, using KEGG analysis, several pathways were enriched, such as insulin secretion, Fc gamma R-mediated phagocytosis, lysine degradation, neuroactive ligand-receptor interaction, serotonergic synapse, bacterial invasion of epithelial cells, hepatocellular carcinoma, RNA degradation, non-small-cell lung cancer, Cushing's syndrome, and EGFR tyrosine kinase inhibitor resistance. (Fig. 4C and Table SII). These results provided the potential regulatory cellular pathways by which the differential circRNAs may be involved in the development of ROP.
To fully understand the possible mechanisms and signals involved, 9,756 dysregulated mRNAs were identified using the same cDNA libraries constructed and sequenced above, which included 4,760 upregulated and 4,996 downregulated mRNAs compared to those in the control. The differentially expressed mRNAs with a $\mathrm{FC}>2$ and $\mathrm{P}<0.05$ were selected for GO analysis. As shown in Fig. S1A and Table III, the results indicated that angiogenesis ranked in the top 5 upregulated GO terms that were significantly enriched for biological processes; this coincided with the findings of pathological angiogenesis observed in the OIR mouse retinas (Fig. 1). Therefore, the coregulation network of mRNA-circRNA-miRNA associated with angiogenesis in ROP was examined, and 77 differential mRNAs that were 
A

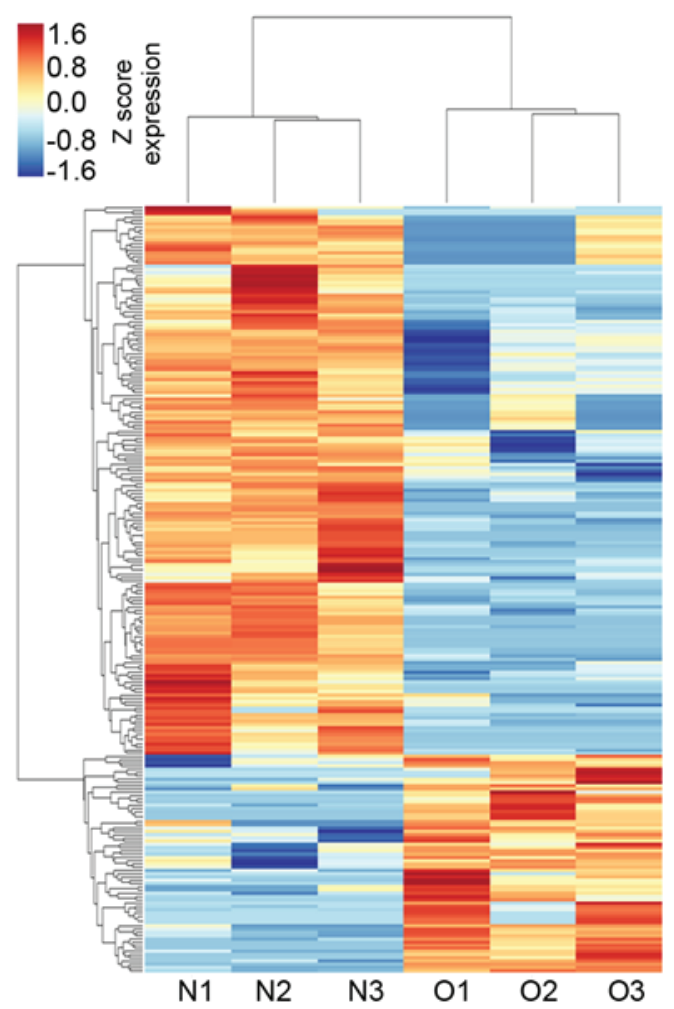

C

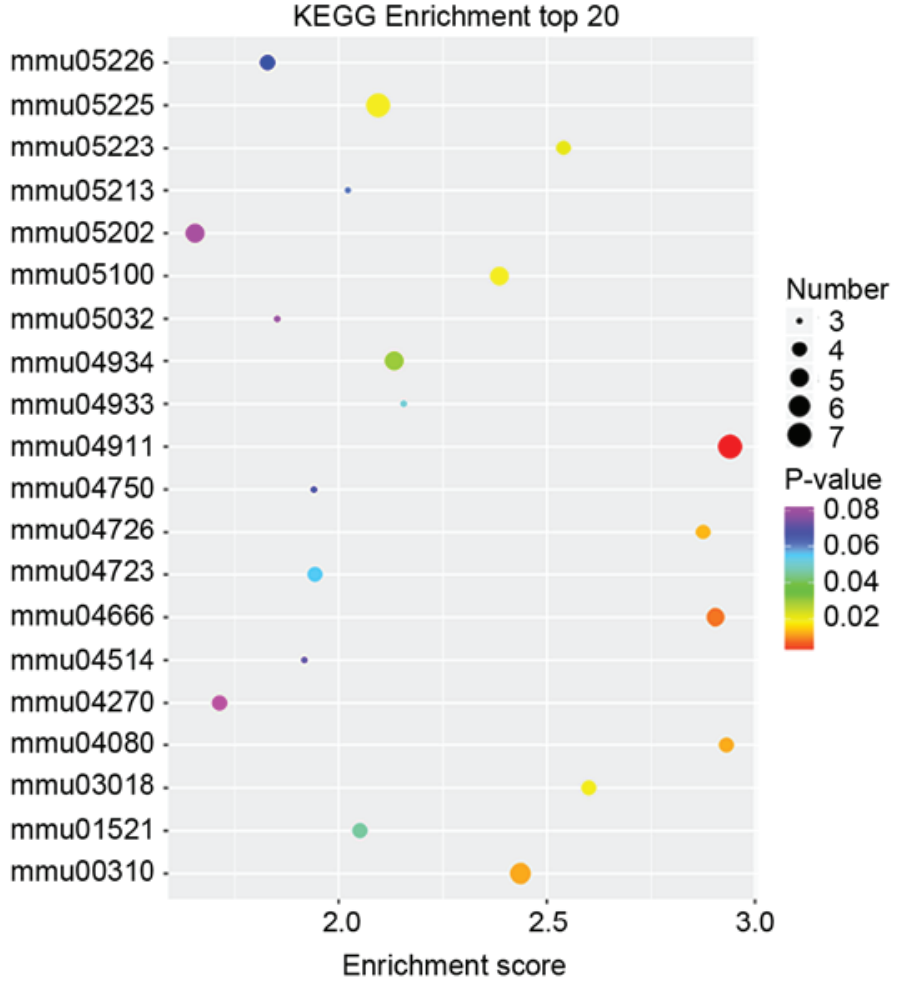

B

\section{Category}

Biological process Cellular component Molecular function

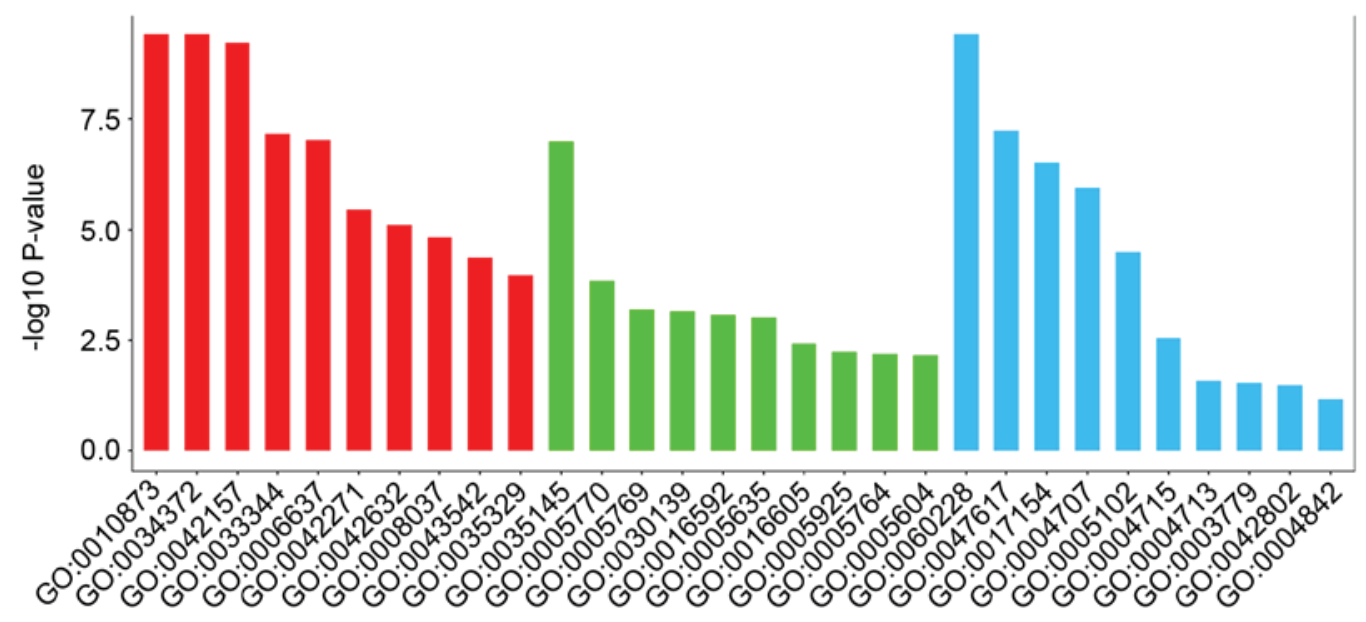

Figure 4. Functional prediction of differential circRNAs in OIR retinas. (A) Hierarchical cluster of the circRNAs that were differentially expressed in the control and OIR retinas. The $\log 2$ value ranges from -2.0 to 2.0 are shown as shades of red or blue according to the key, and P $<0.05$ was used as the cut-off. Rows represent the individual probe set, and the columns represent the experimental samples. N1-3, sample 1-3 from the control group and O1-3, sample 1-3 from the OIR group. (B) Top 10 upregulated GO terms of all the differential circRNA transcripts listed according to their P-values. (C) Top 20 KEGG predictions for differential circRNAs based on their P-values, and the homologous coding genes from the same genomic loci of the predicted differential circRNAs are shown. The $\mathrm{x}$-axis represents the enrichment score. The size of the bubble indicates the number of enriched circRNAs: The larger the bubble, the greater the number of circRNAs. The variation of colors from purple to red represents the P-values for the enrichment analysis. circRNAs, circulating RNAs; OIR, oxygen-induced retinopathy; KEGG, Kyoto Encyclopedia of Genes and Genomes; GO, Gene Ontology.

enriched in the biological process of angiogenesis were chosen for the following analysis (Fig. S1B).

Prediction and identification of differential miRNAs in the OIR model. In Small RNA sequencing, 14 differentially expressed miRNAs (nine known and five novel miRNAs) were identified. Of these nine known differential miRNAs (Table IV), five miRNAs were upregulated (Fig. 5A), while other four miRNAs including mmu-let-7k, mmu-miR-203-3p,
mmu-miR-412-5p and mmu-miR-5128 were downregulated in the OIR samples.

Potential regulatory roles of circRNAs serving as ceRNA via circRNA-miRNA-mRNA coregulation in the OIR model. CircRNAs may contain sequences with a high level of homology to linear coding RNAs such as mRNAs. By competing with an mRNA for the same miRNA reaction element (MREs), circRNAs regulate the activity of mRNAs by forming a large 
Table II. Differentially expressed circulating RNA transcripts associated with the top 10 upregulated GO terms in oxygeninduced retinopathy retinas.

\begin{tabular}{ll} 
A, Biological process & \\
\hline ID & \\
\hline GO:0010873 & \multicolumn{1}{c}{ Term } \\
& $\begin{array}{l}\text { Positive regulation of cholesterol } \\
\text { Esterification }\end{array}$ \\
GO:0034372 & $\begin{array}{l}\text { Very-low-density lipoprotein } \\
\text { particle remodeling }\end{array}$ \\
GO:0042157 & $\begin{array}{l}\text { Lipoprotein metabolic process } \\
\text { Cholesterol efflux }\end{array}$ \\
GO:0033344 & $\begin{array}{l}\text { Acyl-CoA metabolic process } \\
\text { Susceptibility to natural killer cell } \\
\text { GO:0006637 }\end{array}$ \\
GO:0042271 & $\begin{array}{l}\text { Cholesterol homeostasis } \\
\text { Cell recognition }\end{array}$ \\
GO:0042632 & Endothelial cell migration \\
GO:0008037 & Hippo signaling \\
GO:0043542 & \\
GO:0035329 &
\end{tabular}

B, Cellular component

ID

\begin{tabular}{ll}
\hline GO:0035145 & Exon-exon junction complex \\
GO:0005770 & Late endosome \\
GO:0005769 & Early endosome \\
GO:0030139 & Endocytic vesicle \\
GO:0016592 & Mediator complex \\
GO:0005635 & Nuclear envelope \\
GO:0016605 & PML body \\
GO:0005925 & Focal adhesion \\
GO:0005764 & Lysosome \\
GO:0005604 & Basement membrane \\
\hline
\end{tabular}

C, Molecular function

\begin{tabular}{|c|c|}
\hline ID & Term \\
\hline GO:0060228 & $\begin{array}{l}\text { Phosphatidylcholine-sterol } \\
\text { O-acyltransferase activator activity }\end{array}$ \\
\hline GO:0047617 & Acyl-CoA hydrolase activity \\
\hline GO:0017154 & Semaphorin receptor activity \\
\hline GO:0004707 & MAP kinase activity \\
\hline GO:0005102 & Signaling receptor binding \\
\hline GO:0004715 & $\begin{array}{l}\text { Non-membrane spanning protein } \\
\text { tyrosine kinase activity }\end{array}$ \\
\hline GO:0004713 & Protein tyrosine kinase activity \\
\hline GO:0003779 & Actin binding \\
\hline GO:0042802 & Identical protein binding \\
\hline GO:0004842 & $\begin{array}{l}\text { Ubiquitin-protein transferase } \\
\text { activity }\end{array}$ \\
\hline
\end{tabular}

GO, Gene Ontology.
Table III. Top 10 upregulated GO terms enriched for biological process of all differential mRNAs in oxygen-induced retinopathy retinas.

\begin{tabular}{ll} 
ID & \multicolumn{1}{c}{ Term } \\
\hline GO:1990744 & $\begin{array}{l}\text { Primary miRNA methylation } \\
\text { Regulation of chaperone-mediated } \\
\text { autophagy } \\
\text { Positive regulation of alpha-beta } \\
\text { T cell activation } \\
\text { GO:0046635 }\end{array}$ \\
Positive regulation of single stranded \\
viral RNA replication via double \\
stranded DNA intermediate \\
Angiogenesis \\
GO:0001525 & Cell cycle \\
GO:0007049 & Protein ubiquitination \\
GO:0016567 & Cell division \\
GO:0051301 & Cilium assembly \\
GO:0060271 & mRNA methylation \\
GO:0080009 &
\end{tabular}

miRNA, microRNA; GO, Gene Ontology.

Table IV. Differential miRNAs in oxygen-induced retinopathy retinas.

\begin{tabular}{lll} 
A, Downregulated & & \\
\hline miRNA_ID & FC value & P-value \\
\hline mmu-let-7k & 0.414673 & $0.030135^{\mathrm{a}}$ \\
mmu-miR-203-3p & 0.450087 & $0.03282^{\mathrm{a}}$ \\
mmu-miR-412-5p & 0.516986 & $0.042373^{\mathrm{a}}$ \\
mmu-miR-5128 & 0.122681 & $1.06 \times 10^{-5 c}$
\end{tabular}

B, Upregulated

\begin{tabular}{lll}
\hline miRNA_ID & FC value & P-value \\
\hline mmu-miR-126a-3p & 1.754258 & $0.047684^{\mathrm{a}}$ \\
mmu-miR-143-3p & 2.278029 & $0.00154^{\mathrm{b}}$ \\
mmu-miR-146a-5p & 2.452372 & $0.009801^{\mathrm{b}}$ \\
mmu-miR-210-3p & 2.317902 & $0.02668^{\mathrm{a}}$ \\
mmu-miR-503-3p & 2.733501 & $0.018975^{\mathrm{a}}$ \\
\hline
\end{tabular}

${ }^{\mathrm{a}} \mathrm{P}<0.05,{ }^{\mathrm{b}} \mathrm{P}<0.01,{ }^{\mathrm{c}} \mathrm{P}<0.001$. miRNA, microRNA; FC, fold change.

ceRNA network. In the present study, 77 upregulated mRNAs that were enriched for the biological process of angiogenesis and 39 circRNAs (Fig. 5B) were separately subjected to the target prediction of four downregulated miRNAs using miRanda (43). For example, mmu_circ_0000668 was predicted to react with mmu-miR-203-3p via two MREs (Fig. 5C). Using a correlation coefficient $\geq 0.7$ and $\mathrm{P} \leq 0.05$ as a cut-off, correlation analysis was performed for circRNA-mRNA regulatory pair prediction. 
A

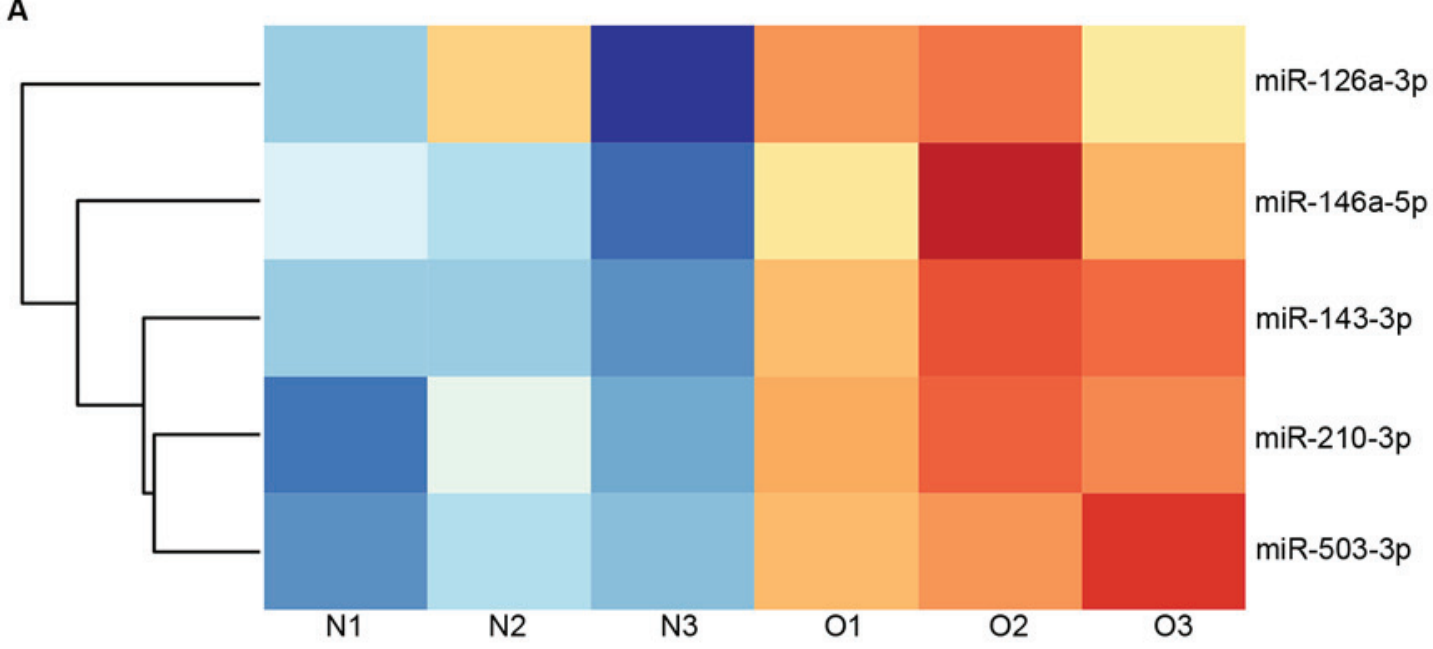

B

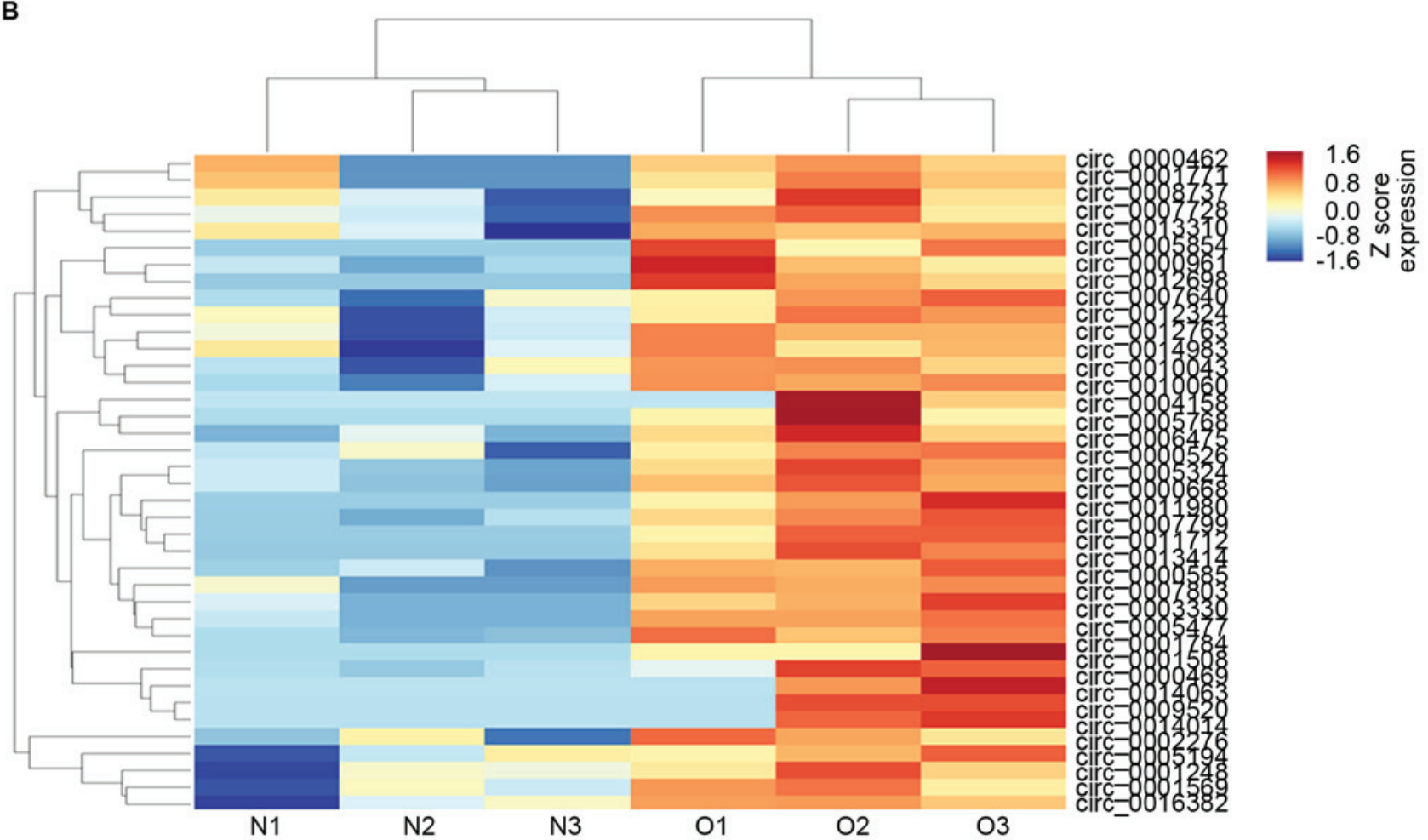

C

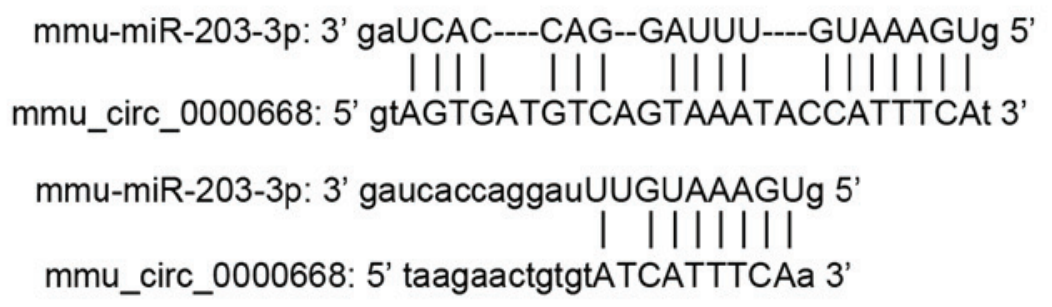

Figure 5. circRNA-miRNA-mRNA coregulatory network was constructed using hypergeometric distribution analysis. (A) Hierarchical cluster analysis of upregulated miRNAs in OIR retinas. $\log 2$ values from -2.0 to 2.0 and $\mathrm{P}<0.05$ was used as the cut-off. (B) Heatmap of the differential circRNAs that contain the miRNA-response elements of downregulated miRNAs and those that were co-expressed with angiogenesis-associated differentially expressed mRNAs in OIR retinas are shown. N1-3 represents samples 1-3 from the control group and O1-3 represents samples 1-3 from the OIR group. (C) Prediction of the complementary region of mmu_circ_0000668 for the seeding sequence of mmu-miR-203-3p was performed using miRanda. circRNA, circulating RNA; $\mathrm{miRNA} / \mathrm{miR}$, microRNA; OIR, oxygen-induced retinopathy.

A total of 9 circRNAs, including mmu_circ_0005477,mmu_ circ_0013414, mmu_circ_0012698, mmu_circ_0000668, mmu_circ_0007799,mmu_circ_0005324,mmu_circ_0008737, mmu_circ_0005768 and mmu_circ_0004158 were predicted as ceRNAs, and the ceRNETs were constructed. As shown in Fig. 6A, 4 ceRNAs (mmu_circ_0005477, mmu_circ_0013414, 


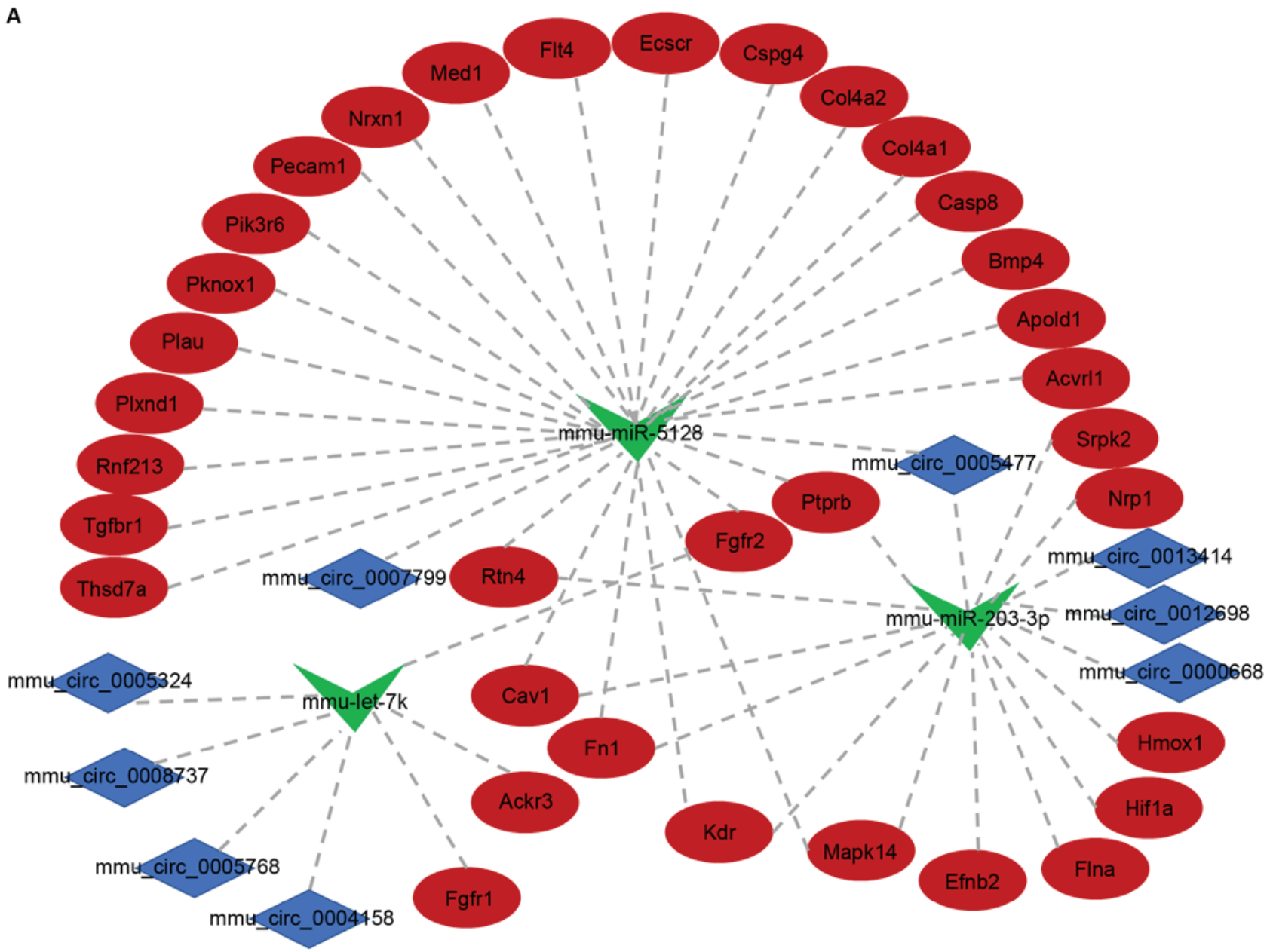

B

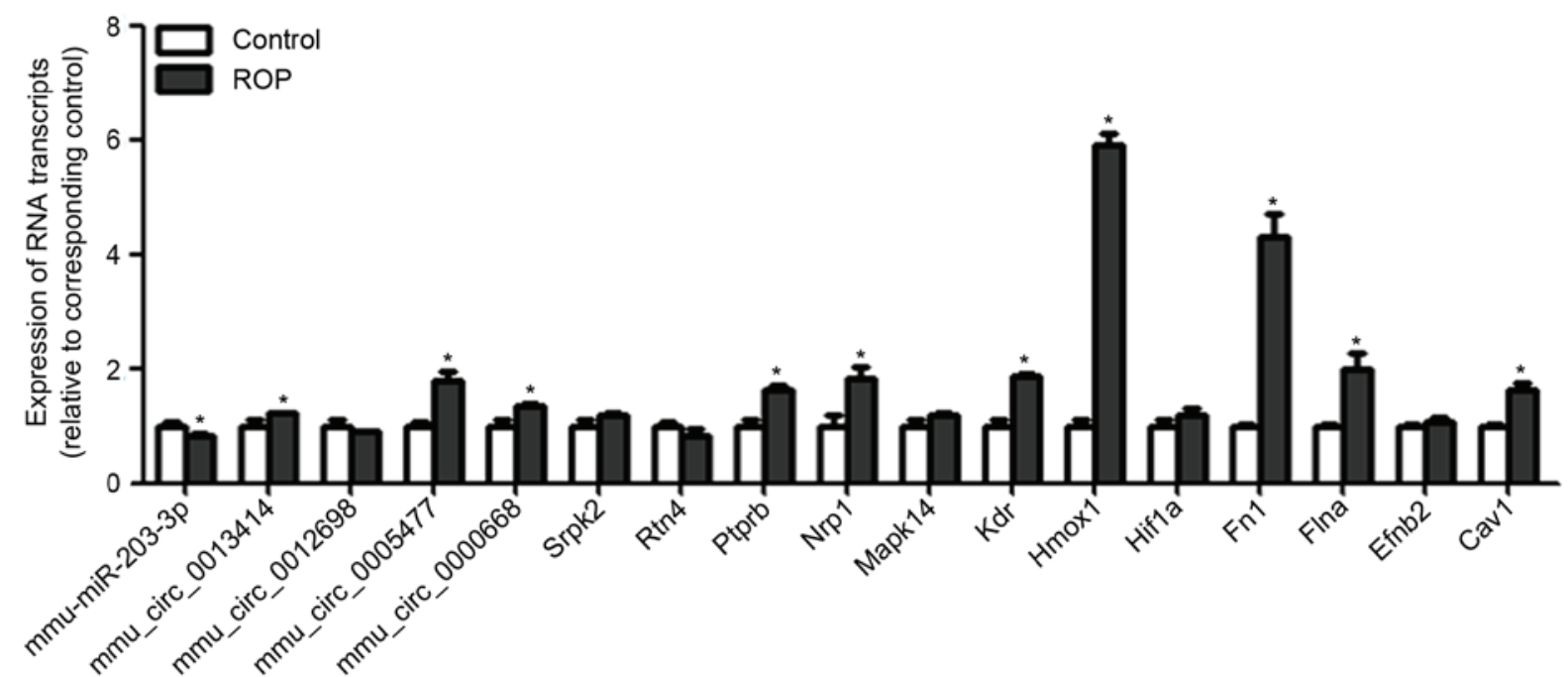

Figure 6. Validation of the circRNA-miRNA-mRNA coregulatory network. (A) circRNA-miRNA-mRNA network associated with angiogenesis. The blue, green, and red nodes represent the circRNA, miRNA and mRNA, respectively. (B) Validation of the selected circRNAs, miRNAs and mRNAs of the coregulation network was performed using reverse-transcription-quantitative PCR. ${ }^{~} \mathrm{P}<0.05$ vs. respective control. circRNA, circulating RNA; miRNA, microRNA.

mmu_circ_0012698 and mmu_circ_0000668) were predicted to regulate the expression of mRNAs, such as SRSF protein kinase 2 (Srpk2), neuropilin 1 (Nrp1), heme oxygenase 1 (Hmox1), hypoxia-inducible factor 1 subunit alpha (Hifla), filamin alpha (Flna), ephrin B2 (Efnb2), mitogen-activated protein kinase 14 (Mapk14), kinase insert domain receptor (Kdr), fibronectin 1 (Fn1), caveolin 1 (Cav1), reticulon 4 (Rtn4), and protein tyrosine phosphatase receptor type B (Ptprb) by competing with mmu-miR-203-3p.

In addition, the regulation of fibroblast growth factor receptor (Fgfr)1, Fgfr2, and atypical chemokine receptor 3 by mmu-let-7k may be modulated by mmu_circ_0005324, $\mathrm{mmu}$ _circ_0008737, $\mathrm{mmu}$ _circ_0005768 and $\mathrm{mmu}$ circ_0004158, which possess the MREs of let-7k. These 
results suggest that $\mathrm{mmu}$ _circ_0005477 may capture mmu-miR-203-3p but also mmu-miR-5128, which target 19 angiogenesis-associated coding genes. Certain RNA transcripts involved in angiogenesis-associated ceRNETs (such as mmu-miR-203-3p-associated) were further verified using RT-qPCR assay as shown in Fig. 6B and were primarily consistent with the sequencing results. In the OIR model, mmu-miR-203-3p was significantly decreased, whereas the expression of mmu_circ_0013414, mmu_circ_0005477 and mmu_circ_0000668 were significantly increased. In addition, the mRNAs expression levels of Ptprb, Nrp1, Kdr, Hmox1, Fn1, Flna and Cav1 were also significantly upregulated (Fig. 6B).

\section{Discussion}

At present, ROP is the leading cause of childhood blindness. However, the underlying mechanisms are not fully understood. Dysregulated circRNA expression has been frequently reported in human diseases, including in multiple ocular disorders $(22,26,44,45)$. In the OIR model, a widely utilized mouse model for ROP, neonatal mice were exposed to $75 \%$ oxygen between P7 and P12, and the hyperoxia caused vessel regression in the central retina and the cessation of normal radial vessel growth (46). The neonatal mice were returned to normal air between P12 and P17, the period during which vascular regrowth and neovascularization is maximal (6). The OIR and control mouse retinas on P17 were chosen in the present study to determine the expression profiles of circRNAs and mRNAs via high-throughput transcriptome sequencing analysis. A total of 27,878 circRNAs were identified, including 236 differentially expressed circRNAs, as well as 9,756 differentially expressed mRNAs between the OIR and control groups. Of the predicted circRNAs, $>70 \%$ were $<1,000$ bp in length, $68.4 \%$ had 5 or fewer exons and $27.9 \%$ were identified as known circRNAs according to the circBase database. These characteristics matched those of the known characteristics of circRNAs (22), suggesting the reliability of these identified circRNAs. The sequencing results made it possible to determine the regulatory roles of circRNAs in ROP development.

Whole-transcriptome sequencing has been recognized as a more valuable methodology than microarray hybridization as it provides annotation of genes/genomes for the reconstruction of genetic interaction networks that are associated with certain cellular functions, growth/development and biological systems (47). In the present study, 9,756 differentially expressed mRNAs and the 236 circRNAs underwent functional annotation and were found to be enriched in biological processes such as primary miRNA methylation, the regulation of chaperone-mediated autophagy, the positive regulation of $\alpha-\beta$ T cell activation, the positive regulation of single-stranded viral RNA replication via a double-stranded DNA intermediate and angiogenesis. Angiogenesis ranked in the top 5 upregulated GO terms, in agreement with the well-established development of ROP that is characterized by abnormal angiogenesis (7). During ROP, development of the physiological retinal vasculature is delayed, followed by abnormal retinal neovascularization (7). As a result, pathological angiogenesis leads to retinal fibrosis, detachment and blindness $(7,48)$. These results suggest a possible regulatory mechanism of circRNA, by which, the circRNAs interact with the mRNAs and modulate angiogenesis during ROP. Therefore, a comprehensive understanding of the circRNA-mRNA network may be required to better understand the underlying mechanisms during ROP in addition to the known molecular mechanisms.

Previously, the ceRNA hypothesis has provided a novel angle to interpret RNA-RNA interactions (49). Functional RNA molecules are silenced by miRNA-mediated miRNA-mRNA interactions through sequence-specific binding sites. According to this hypothesis, IncRNAs, circRNAs, pseudogenes, or other mRNAs that contain the same miRNA binding sites are able to competitively bind to miRNA, and, in turn, this regulates the functional activity of the RNAs that are targeted by the now sequestered miRNA molecules (49). As a novel class of noncoding RNAs, circRNAs are usually generated from protein-coding genes by alternative splicing, which can form a covalent ring structure (50), and $>80 \%$ of circRNAs contain the same sequences as their homologous protein-coding mRNAs or exons, enabling them to serve as ceRNAs by absorbing the miRNAs $(49,50)$.

In the present study, 9 circRNAs were predicted to be ceRNAs that regulate angiogenesis-associated coding genes in ROP by competing with miR-203-3p, miR-5128, and let-7k. Of the three differentially expressed miRNAs, multiple studies have shown that miR-203-3p serves a critical role in the development and progression of a number of different types of tumor (51-55) and may regulate placental angiogenesis through inhibiting the expression of VEGFA and VEGFR2 (56). In ocular diseases, miR-203-3p was reported to serve as a novel regulator of zebrafish retina regeneration by repressing pax6b, which is necessary for progenitor cell expansion (57). Additionally, miR-203-3p is thought to be involved in the retinal differentiation of amniotic epithelial stem cells and umbilical cord blood-derived mesenchymal stem cells, possibly by targeting multiple retina development-relevant genes, such as dickkopf WNT signaling pathway inhibitor 1, cone-rod homeobox, RAR-related orphan receptor beta, neurogenic differentiation 1 , neural retina leucine zipper and thyroid hormone receptor beta (58). The results of the present study suggest that the upregulated expression of angiogenesis-associated mRNAs, such as Hmox1 (59), Fn1 (60), Flna (61), Nrp1 (62), Kdr (63), Cav1 (64), Ptprb (65), Srpk2 (66), Hif1a (67), Efnb2 (68) and Mapk14 (69), which were predicted to be targets of miR-203-3p, may be regulated by circ_0005477, circ_0013414, circ_0012698 and circ_0000668 in ROP retinas. The bioinformatics results were in accordance with the previous reports where it was determined that miR-203 targets Hifla and tenascin C-enriched ECM to regulate glioblastoma aggression (70) and suppresses the expression level of Cav1 mRNA in melanoma cells (71). Liu et al (72) demonstrated that circRNA-ZNF609 silencing ameliorates vascular endothelial dysfunction through a cZNF609/miR-615-5p/MEF2A signaling network, and that circular RNA-HIPK3 mediates retinal vascular dysfunction in diabetes mellitus by acting as an endogenous miR-30a-3p sponge (73). However, the expression profiles and functions of circRNAs in ROP has not been reported, to the best of our knowledge. The present study is the first to identify a novel molecular mechanism that involves the circRNA-miRNA-mRNA interaction in regulating the development of ROP. Hypoxia-induced angiogenesis serves a central role in the pathogenesis of ROP (74), which is also 
critical for tumor progression, vascularization and metastasis in cancer (75); therefore, ROP and cancer may share common pathogenic mechanisms (76). Considering other time points (e.g. P12 and P21) are also vital for the development of OIR, the present study primarily focused on the differential circRNAs and related regulatory networks involved in angiogenesis during OIR on P17, which may have at least provided novel targets for proliferative retinopathies and possibly for human malignancies.

In conclusion, the present study identified a panel of circRNAs that were differentially expressed in ROP, analyzed the potential biological functions and pathways that may be regulated by these differential circRNAs and predicted the circRNA-miRNA interaction pairs. Furthermore, a circRNA-miRNA-mRNA interaction network was determined. circRNAs may serve a novel regulatory role as a ceRNA, which is involved in the regulation of abnormal angiogenesis during the development of ROP. Nevertheless, detailed functional studies are required to comprehensively validate the results and elucidate the potential molecular mechanisms of competing endogenous circRNAs in ROP.

\section{Acknowledgements}

The authors would like to thank Dr Qidong $\mathrm{Zu}$ (OE Biotech, Inc., Shanghai, China, http://www.oebiotech.com/) for assistance with the bioinformatics analysis of the RNA sequencing data.

\section{Funding}

The present study was funded by The National Natural Science Foundation of China (grant nos. 81500733, 81502500, 81501703,81302382 and 81602181), The Natural Science Foundation of Jiangsu Province, China (Jiangsu, China; grant nos. BK20150292, BK20150293 and BK20150294) and Suzhou People's Livelihood Science and Technology Project (Suzhou, China; grant no. SS201648).

\section{Availability of data and materials}

The datasets used and/or analyzed during the present study are available from the author on reasonable request.

\section{Authors' contributions}

The present study was conceived by $\mathrm{HuZ}, \mathrm{KF}, \mathrm{YJ}$ and $\mathrm{HX}$. The data were collected by HS, YW, XL, JL and HeZ, analyzed by HS, YW, XL, JL, HeZ and YJ, and interpreted by HuZ, MT, XJ, LZ, YS, YH and HX. The experiments were performed by MT, $\mathrm{XJ}$ and LZ. The study was managed and coordinated by HX. Resources were provided by HS, YW, XL, JL, HeZ, YS and YH. The study was supervised by YS, YH and KF. The manuscript was written by $\mathrm{HuZ}, \mathrm{YJ}$ and $\mathrm{HX}$, and reviewed and edited by YJ and HX. All authors read and approved the final manuscript.

\section{Ethics approval and consent to participate}

The present study was approved by The Ethics Review Board of The Children's Hospital of Soochow University (Suzhou, China).

\section{Patient consent for publication}

Not applicable.

\section{Competing interests}

The authors declare that they have no competing interests.

\section{References}

1. Hellstrom A, Smith LE and Dammann O: Retinopathy of prematurity. Lancet 382: 1445-1457, 2013.

2. Fielder A, Blencowe H, O'Connor A and Gilbert C: Impact of retinopathy of prematurity on ocular structures and visual functions. Arch Dis Child Fetal Neonatal Ed 100: F179-F184, 2015.

3. Patz A, Hoeck LE and De La Cruz E: Studies on the effect of high oxygen administration in retrolental fibroplasia. I. Nursery observations. Am J Ophthalmol 35: 1248-1253, 1952.

4. Ashton N: Pathological basis of retrolental fibroplasia. Br J Ophthalmol 38: 385-396, 1954.

5. Smith LE, Wesolowski E, McLellan A, Kostyk SK, D'Amato R, Sullivan R and D'Amore PA: Oxygen-induced retinopathy in the mouse. Invest Ophthalmol Vis Sci 35: 101-111, 1994.

6. Connor KM, Krah NM, Dennison RJ, Aderman CM, Chen J, Guerin KI, Sapieha P, Stahl A, Willett KL and Smith LE: Quantification of oxygen-induced retinopathy in the mouse: A model of vessel loss, vessel regrowth and pathological angiogenesis. Nat Protoc 4: 1565-1573, 2009.

7. Hartnett ME and Penn JS: Mechanisms and management of retinopathy of prematurity. N Engl J Med 367: 2515-2526, 2012.

8. Chen J, Connor KM, Aderman CM and Smith LE: Erythropoietin deficiency decreases vascular stability in mice. J Clin Invest 118: 526-533, 2008.

9. Pierce EA, Avery RL, Foley ED, Aiello LP and Smith LE: Vascular endothelial growth factor/vascular permeability factor expression in a mouse model of retinal neovascularization. Proc Natl Acad Sci USA 92: 905-909, 1995.

10. Pierce EA, Foley ED and Smith LE: Regulation of vascular endothelial growth factor by oxygen in a model of retinopathy of prematurity. Arch Ophthalmol 114: 1219-1228, 1996.

11. Solebo AL, Teoh L and Rahi J: Epidemiology of blindness in children. Arch Dis Child 102: 853-857, 2017.

12. Chan-Ling T, Gole GA, Quinn GE, Adamson SJ and Darlow BA: Pathophysiology, screening and treatment of ROP: A multi-disciplinary perspective. Prog Retin Eye Res 62: 77-119, 2018.

13. Sato T, Kusaka S, Hashida N, Saishin Y, Fujikado T and Tano Y: Comprehensive gene-expression profile in murine oxygen-induced retinopathy. Br J Ophthalmol 93: 96-103, 2009.

14. Wang Y, Wu S, Yang Y, Peng F, Li Q, Tian P, Xiang E, Liang H, Wang B, Zhou X, et al: Differentially expressed miRNAs in oxygeninduced retinopathy newborn mouse models. Mol Med Rep 15: 146-152, 2017.

15. Shen J, Yang X, Xie B, Chen Y, Swaim M, Hackett SF and Campochiaro PA: MicroRNAs regulate ocular neovascularization. Mol Ther 16: 1208-1216, 2008.

16. Bai Y, Bai X, Wang Z, Zhang X, Ruan C and Miao J: MicroRNA-126 inhibits ischemia-induced retinal neovascularization via regulating angiogenic growth factors. Exp Mol Pathol 91: 471-477, 2011.

17. Tea M, Michael MZ, Brereton HM and Williams KA: Stability of small non-coding RNA reference gene expression in the rat retina during exposure to cyclic hyperoxia. Mol Vis 19: 501-508, 2013.

18. Zhao R, Hou W, Zhang Z, Qian L and Jiang L: Differential Expression of Mir-1 26 and Vascular endothelial growth factor in retinal cells of metabolic acidosis-induced neonatal rats. J Nanosci Nanotechnol 15: 2088-2093, 2015.

19. Zhao R, Qian L and Jiang L: miRNA-dependent cross-talk between VEGF and Ang-2 in hypoxia-induced microvascular dysfunction. Biochem Biophys Res Commun 452: 428-435, 2014.

20. Nunes DN, Dias-Neto E, Cardo-Vila M, Edwards JK, Dobroff AS, Giordano RJ, Mandelin J, Brentani HP, Hasselgren C, Yao VJ, et al: Synchronous down-modulation of miR-17 family members is an early causative event in the retinal angiogenic switch. Proc Natl Acad Sci USA 112: 3770-3775, 2015. 
21. Chen XK, Ouyang LJ, Yin ZQ, Xia YY, Chen XR, Shi H, Xiong Y and Pi LH: Effects of microRNA-29a on retinopathy of prematurity by targeting AGT in a mouse model. Am J Transl Res 9: 791-801, 2017.

22. Memczak S, Jens M, Elefsinioti A, Torti F, Krueger J, Rybak A, Maier L, Mackowiak SD, Gregersen LH, Munschauer M, et al: Circular RNAs are a large class of animal RNAs with regulatory potency. Nature 495: 333-338, 2013

23. Fischer JW and Leung AK: CircRNAs: A regulator of cellular stress. Crit Rev Biochem Mol Biol 52: 220-233, 2017.

24. Han J, Gao L, Dong J, Bai J, Zhang M and Zheng J: The expression profile of developmental stage-dependent circular RNA in the immature rat retina. Mol Vis 23: 457-469, 2017.

25. Singh M, George AK, Homme RP, Majumder A, Laha A Sandhu HS and Tyagi SC: Circular RNAs profiling in the cystathionine-beta-synthase mutant mouse reveals novel gene targets for hyperhomocysteinemia induced ocular disorders. Exp Eye Res 174: 80-92, 2018

26. Luo Y, Liu S and Yao K: Transcriptome-wide Investigation of mRNA/circRNA in miR-184 and Its $r .57 \mathrm{c}>\mathrm{u}$ mutant type treatment of human lens epithelial cells. Mol Ther Nucleic Acids 7: 71-80, 2017.

27. Xu H, Zhang L, Gu L, Gao G, Li W, Xu G, Wang J, Gao F, Xu JY, Yao J, et al: Subretinal delivery of AAV2-mediated human erythropoietin gene is protective and safe in experimental diabetic retinopathy. Invest Ophthalmol Vis Sci 55: 1519-1530, 2014.

28. Zhu KP,Zhang CL, Ma XL, Hu JP, Cai T and Zhang L: Analyzing the interactions of mRNAs and ncRNAs to predict competing endogenous RNA networks in osteosarcoma chemo-resistance. Mol Ther 27: 518-530, 2019.

29. Gao Y, Wang J and Zhao F: CIRI: An efficient and unbiased algorithm for de novo circular RNA identification. Genome Biol 16: 4, 2015

30. Glazar P, Papavasileiou P and Rajewsky N: circBase: A database for circular RNAs. Rna 20: 1666-1670, 2014

31. Anders S and Huber W: Differential expression analysis for sequence count data. Genome Biol 11: R106, 2010

32. Huang da W, Sherman BT and Lempicki RA: Systematic and integrative analysis of large gene lists using DAVID bioinformatics resources. Nat Pro 4: 44-57, 2009.

33. Kanehisa M, Araki M, Goto S, Hattori M, Hirakawa M, Itoh M, Katayama T, Kawashima S, Okuda S, Tokimatsu T and Yamanishi Y: KEGG for linking genomes to life and the environment. Nucleic Acids Res 36: D480-484, 2008.

34. John B, Enright AJ, Aravin A, Tuschl T, Sander C and Marks DS: Human MicroRNA targets. PLoS Biol 2: e363, 2004.

35. R Core Team (2012). R: A language and environment for statistical computing. R Foundation for Statistical Computing, Vienna, Austria. ISBN 3-900051-07-0.

36. Griffiths-Jones S, Bateman A, Marshall M, Khanna A and Eddy SR: Rfam: An RNA family database. Nucleic Acids Res 31: 439-441, 2003

37. Griffiths-Jones S, Saini HK, van Dongen S and Enright AJ: miRBase: Tools for microRNA genomics. Nucleic Acids Res 36: D154-158, 2008

38. Friedlander MR, Mackowiak SD, Li N, Chen W and Rajewsky N: miRDeep2 accurately identifies known and hundreds of novel microRNA genes in seven animal clades. Nucleic Acids Res 40: 37-52, 2012.

39. Sun L, Luo H, Bu D, Zhao G, Yu K, Zhang C, Liu Y, Chen R and Zhao Y: Utilizing sequence intrinsic composition to classify protein-coding and long non-coding transcripts. Nucleic Acids Res 41: e166, 2013

40. Nie J, Zhang J, Wang L, Lu L, Yuan Q, An F, Zhang S and Jiao Y: Adipocytes promote cholangiocarcinoma metastasis through fatty acid binding protein 4 . J Exp Clin Cancer Res 36: 183,2017

41. Livak KJ and Schmittgen TD: Analysis of relative gene expression data using real-time quantitative PCR and the 2(-Delta Delta C(T)) method. Methods 25: 402-408, 2001.

42. Reynaud X and Dorey CK: Extraretinal neovascularization induced by hypoxic episodes in the neonatal rat. Invest Ophthalmol Vis Sci 35: 3169-3177, 1994.

43. Enright AJ, John B, Gaul U, Tuschl T, Sander C and Marks DS MicroRNA targets in Drosophila. Genome Biol 5: R1, 2003.

44. Zhang SJ, Chen X, Li CP, Li XM, Liu C, Liu BH, Shan K, Jiang Q, Zhao C and Yan B: Identification and characterization of circular RNAs as a new class of putative biomarkers in diabetes retinopathy. Invest Ophthalmol Vis Sci 58: 6500-6509, 2017.
45. Gu Y, Ke G, Wang L, Zhou E, Zhu K and Wei Y: Altered expression profile of circular RNAs in the serum of patients with diabetic retinopathy revealed by microarray. Ophthalmic Res 58: 176-184, 2017.

46. Ashton N: Animal experiments in retrolental fibroplasia. Trans Am Acad Ophthalmol Otolaryngol 58: 51-54, 1954.

47. Jiang Z, Zhou X, Li R, Michal JJ, Zhang S, Dodson MV, Zhang $\mathrm{Z}$ and Harland RM: Whole transcriptome analysis with sequencing: Methods, challenges and potential solutions. Cell Mol Life Sci 72: 3425-3439, 2015.

48. Mammoto A, Connor KM, Mammoto T, Yung CW, Huh D, Aderman CM, Mostoslavsky G, Smith LE and Ingber DE: A mechanosensitive transcriptional mechanism that controls angiogenesis. Nature 457: 1103-1108, 2009.

49. Tay Y, Rinn J and Pandolfi PP: The multilayered complexity of ceRNA crosstalk and competition. Nature 505: 344-352, 2014.

50. Hansen TB, Jensen TI, Clausen BH, Bramsen JB, Finsen B, Damgaard CK and Kjems J: Natural RNA circles function as efficient microRNA sponges. Nature 495: 384-388, 2013.

51. Zhu X, Er K, Mao C, Yan Q, Xu H, Zhang Y,Zhu J, Cui F, Zhao W and Shi H: miR-203 suppresses tumor growth and angiogenesis by targeting VEGFA in cervical cancer. Cell Physiol Biochem 32: 64-73, 2013.

52. Wang N, Liang H, Zhou Y, Wang C, Zhang S, Pan Y, Wang Y, Yan X, Zhang J, Zhang CY, et al: miR-203 suppresses the proliferation and migration and promotes the apoptosis of lung cancer cells by targeting SRC. PLoS One 9: e105570, 2014.

53. Tian X, Tao F, Zhang B, Dong JT and Zhang Z: The miR-203/SNAI2 axis regulates prostate tumor growth, migration, angiogenesis and stemness potentially by modulating GSK-3beta/beta-CATENIN signal pathway. IUBMB Life 70: 224-236, 2018

54. Lohcharoenkal W, Harada M, Loven J, Meisgen F, Landén NX, Zhang L, Lapins J, Mahapatra KD, Shi H, Nissinen L, et al: MicroRNA-203 inversely correlates with differentiation grade, targets c-MYC, and functions as a tumor suppressor in cSCC. J Invest Dermatol 136: 2485-2494, 2016.

55. Lohcharoenkal W, Das Mahapatra K, Pasquali L, Crudden C, Kular L, Akkaya Ulum YZ, Zhang L, Xu Landén N, Girnita L, Jagodic M, et al: Genome-wide screen for MicroRNAs reveals a role for miR-203 in melanoma metastasis. J Invest Dermatol 138: 882-892, 2018

56. Liu F, Wu W, Wu K, Chen Y, Wu H, Wang $\mathrm{H}$ and Zhang W: MiR-203 participates in human placental angiogenesis by inhibiting VEGFA and VEGFR2 expression. Reprod Sci 25: 358-365, 2018.

57. Rajaram K, Harding RL, Hyde DR and Patton JG: miR-203 regulates progenitor cell proliferation during adult zebrafish retina regeneration. Dev Biol 392: 393-403, 2014.

58. Choi SW, Shin JH, Kim JJ, Shin TH, Seo Y, Kim HS and Kang KS: Direct cell fate conversion of human somatic stem cells into cone and rod photoreceptor-like cells by inhibition of microRNA-203. Oncotarget 7: 42139-42149, 2016.

59. Shanab AY, Elshaer SL, El-Azab MF, Soliman S, Sabbineni H, Matragoon S, Fagan SC and El-Remessy AB: Candesartan stimulates reparative angiogenesis in ischemic retinopathy model: Role of hemeoxygenase-1 (HO-1). Angiogenesis 18: 137-150, 2015

60. Soikkeli J, Podlasz P, Yin M, Nummela P, Jahkola T, Virolainen S, Krogerus L, Heikkilä P, von Smitten K, Saksela O and Hölttä E: Metastatic outgrowth encompasses COL-I, FN1, and POSTN up-regulation and assembly to fibrillar networks regulating cell adhesion, migration, and growth. Am J Pathol 177: 387-403, 2010.

61. Segura I, Lange C, Knevels E, Moskalyuk A, Pulizzi R, Eelen G, Chaze T, Tudor C, Boulegue C, Holt M, et al: The Oxygen Sensor PHD2 controls dendritic spines and synapses via modification of filamin A. Cell Rep 14: 2653-2667, 2016.

62. Raimondi C, Brash JT, Fantin A and Ruhrberg C: NRP1 function and targeting in neurovascular development and eye disease. Prog Retin Eye Res 52: 64-83, 2016

63. McLeod DS, Taomoto M, Cao J, Zhu Z, Witte L and Lutty GA Localization of VEGF receptor-2 (KDR/Flk-1) and effects of blocking it in oxygen-induced retinopathy. Invest Ophthalmol Vis Sci 43: 474-482, 2002.

64. Tu F, Pang Q, Chen X, Huang T, Liu M and Zhai Q: Angiogenic effects of apigenin on endothelial cells after hypoxia-reoxygenation via the caveolin-1 pathway. Int J Mol Med 40: 1639-1648, 2017. 
65. Behjati S, Tarpey PS, Sheldon H, Martincorena I, Van Loo P, Gundem G, Wedge DC, Ramakrishna M, Cooke SL, Pillay $\mathrm{N}$, et al: Recurrent PTPRB and PLCG1 mutations in angiosarcoma. Nat Genet 46: 376-379, 2014.

66. Jakubauskiene E, Vilys L, Makino Y, Poellinger L and Kanopka A: Increased serine-arginine (SR) protein phosphorylation changes Pre-mRNA splicing in hypoxia. J Biol Chem 290: 18079-18089, 2015.

67. Peet DJ, Kittipassorn T, Wood JP, Chidlow G and Casson RJ: HIF signalling: The eyes have it. Exp Cell Res 356: 136-140, 2017.

68. Lin Y, Jiang W, Ng J, Jina A and Wang RA: Endothelial ephrin-B2 is essential for arterial vasodilation in mice. Microcirculation 21: 578-586, 2014.

69. Rose BA, Yokota T, Chintalgattu V, Ren S, Iruela-Arispe L, Khakoo AY, Minamisawa S and Wang Y: Cardiac myocyte p38alpha kinase regulates angiogenesis via myocyte-endothelial cell cross-talk during stress-induced remodeling in the heart. J Biol Chem 292: 12787-12800, 2017.

70. Miroshnikova YA, Mouw JK, Barnes JM,Pickup MW, Lakins JN, Kim Y, Lobo K, Persson AI, Reis GF, McKnight TR, et al: Tissue mechanics promote IDH1-dependent HIF1alpha-tenascin C feedback to regulate glioblastoma aggression. Nat Cell Biol 18: 1336-1345, 2016

71. Conde-Perez A, Gros G, Longvert C, Pedersen M, Petit V, Aktary Z, Viros A, Gesbert F, Delmas V, Rambow F, et al: A caveolin-dependent and PI3K/AKT-independent role of PTEN in beta-catenin transcriptional activity. Nat Commun 6: 8093, 2015.
72. Liu C, Yao MD, Li CP, Shan K, Yang H, Wang JJ, Liu B, Li XM, Yao J, Jiang Q and Yan B: Silencing of circular RNA-ZNF609 ameliorates vascular endothelial dysfunction. Theranostics 7: 2863-2877, 2017.

73. Shan K, Liu C, Liu BH, Chen X, Dong R, Liu X, Zhang YY, Liu B, Zhang SJ, Wang JJ, et al: Circular noncoding RNA HIPK3 mediates retinal vascular dysfunction in diabetes mellitus. Circulation 136: 1629-1642, 2017.

74. Penn JS, Madan A, Caldwell RB, Bartoli M, Caldwell RW and Hartnett ME: Vascular endothelial growth factor in eye disease. Prog Retin Eye Res 27: 331-371, 2008.

75. Krock BL, Skuli N and Simon MC: Hypoxia-induced angiogenesis: Good and evil. Genes Cancer 2: 1117-1133, 2011.

76. Filippi L, Dal Monte M, Casini G, Daniotti M, Sereni F and Bagnoli P: Infantile hemangiomas, retinopathy of prematurity and cancer: A common pathogenetic role of the $\beta$-adrenergic system. Med Res Rev 35: 619-652, 2015.

This work is licensed under a Creative Commons Attribution-NonCommercial-NoDerivatives 4.0 International (CC BY-NC-ND 4.0) License. 